\title{
Three dimensional transport analysis for ELM control experiments in ITER-similar shape plasmas at low collisionality in DIII-D
}

\author{
O. Schmitz ${ }^{1}$, T.E. Evans ${ }^{2}$, M.E. Fenstermacher ${ }^{3}$, H. Frerichs ${ }^{1}$, \\ M.W. Jakubowski ${ }^{4}$, M.J. Schaffer ${ }^{2}$, A. Wingen ${ }^{5}$, W.P. West $^{2}$, \\ N.H. Brooks ${ }^{2}$, M. Groth ${ }^{3}$ I. Joseph ${ }^{3}$, C.J. Lasnier ${ }^{3}$, R.A. \\ Moyer $^{6}$, H. Stoschus ${ }^{1}$ B. Unterberg ${ }^{1}$, J.G. Watkins ${ }^{7}$, K.H. \\ Burrell $^{2}$, J.S. deGrassie ${ }^{2}$, K.H. Finken ${ }^{1}$, P. Gohil ${ }^{2}$, Y. Feng ${ }^{4}$, \\ M. Lehnen ${ }^{1}$, S. Mordijck ${ }^{6}$, A. Nicolai ${ }^{1}$, T.H. Osborne ${ }^{2}$, \\ D. Reiter ${ }^{1}$, U. Samm ${ }^{1}$, K.H. Spatschek ${ }^{5}$, E.A. Unterberg ${ }^{8}$, \\ R. Wolf ${ }^{4}$ and the DIII-D and TEXTOR Teams \\ ${ }^{1}$ Forschungszentrum Jülich, IEF4-Plasmaphysik, , D-52425 Jülich, Germany \\ ${ }^{2}$ General Atomics, P.O. Box 85608, San Diego, California, 92186-5608 USA \\ ${ }^{3}$ Lawrence Livermore National Laboratory, Livermore, California USA \\ ${ }^{4}$ Max-Planck-Institut für Plasmaphysik, Greifswald \\ ${ }^{5}$ Heinrich-Heine Universität Düsseldorf, ITP I, 40225 Düsseldorf, Germany \\ ${ }^{6}$ University of California-San Diego, La Jolla, California USA \\ ${ }^{7}$ Sandia National Laboratory, Albuquerque, New Mexico USA \\ 8 Oak Ridge Institute for Science Education, Oak Ridge, Tennessee USA \\ E-mail: o.schmitz@fz-juelich.de
}

\begin{abstract}
A study of three-dimensional perturbed magnetic field structures and transport for edge localized mode control experiments with resonant magnetic perturbations at DIII-D is presented. We focus on ITER-Similar Shape plasmas at ITER relevant electron pedestal collisionalities $\nu_{e}^{*} \sim 0.2$. This study is performed in comparison to results from TEXTOR-Dynamic Ergodic Divertor circular limiter plasmas. For both experiments the magnetic field structure is analyzed in the vacuum paradigm - superimposing the external RMP field on the unperturbed equilibrium. At TEXTOR this description holds for normalized poloidal flux $\Psi_{N}>0.7$ without tearing modes driven by the RMP field. For DIII-D H-mode plasmas the validity of this approach still needs to be established. In this paper a method is discussed to diagnose the degree of edge stochastization based on a comparison between modeled magnetic footprints on the divertor targets and experimental data. Clear evidence is presented for the existence of a generic separatrix perturbation causing striation of target particle fluxes. However, heat fluxes into these striations are small. This observation can be explained by accounting for the different heat and particle source locations and the 3D trajectories of the open, perturbed field lines towards the divertor target. Analysis of the transport characteristics filling the perturbed separatrix lobes based on initial EMC3/EIRENE modeling suggests the existence of open field lines connecting the stochastic edge to the target pattern. However, the width and inward most extent of the stochastic layer can not yet be quantified.
\end{abstract}


PACS numbers: 28.52.Lf, 29.85.Fj, 32.70.-n, 52.55.Hc, 52.55.Fa, 52.70.Kz 


\section{Introduction}

Control and mitigation of transient high heat loads expelled by large Type-I Edge Localized Modes (ELMs) [1] is an important task for next step fusion devices like ITER. Extrapolated to the ITER power level, ELMs will - depending on the actual material of the plasma facing components - cause enhanced carbon erosion or tungsten melting, thereby reducing the lifetime of the ITER wall $[2,3,4]$. However, the particle transport driven by ELMs is beneficial, as it facilitates particle inventory control in a quasi steady state fusion plasma like ITER. A modern method with the potential to achieve both reduction of transient heat loads as well as maintaining a controlled level of outward particle transport - is the application of small, edge Resonant Magnetic Perturbations (RMPs). At the DIII-D tokamak Type-I ELM suppression was pioneered by application of an $n=3$ dominant RMP field [5] and investigated in various plasma shapes with averaged triangularity $0.2<\bar{\delta}<0.6$ and at various pedestal electron collisionalities $0.1<\nu_{e}^{*}<3.5$ [6]. Mitigation of Type-I ELMs with $n=1$ and $n=2$ RMP fields was shown also at the JET tokamak [7]. These robust experimental results suggested application of RMPs as an option for ELM control in ITER and led to an actual design review proposing to implement a flexible set of RMP coils into the ITER design [8, 9].

Stochastization of the edge magnetic field is considered as one possible mechanism behind ELM control with RMPs. At DIII-D the field line tracing code TRIP-3D [10] and the RMP spectral analysis code SURFMN [9] were developed by applying the vacuum paradigm. In this approach, the external RMP components are superimposed linearly onto a stationary, axisymmetric equilibrium without any plasma feedback. It was found that for plasmas at low collisionality $\nu_{e}^{*}<0.2$ and for an edge safety factor $q_{95} \sim 3.5$, the calculated overlap of magnetic islands is a good ordering parameter for the ELM size, with ELM suppression correlated with island overlap in the edge region for at least $\Psi_{N}>0.85$ in normalized poloidal flux [11]. This stochastic system needs to be diagnosed to establish the validity of this approach. At TEXTOR-Dynamic Ergodic Divertor (DED) a direct correlation of the stochastic magnetic field modeled in vacuum paradigm to the experiment was revealed $[12,13,14]$. In this paper a method is described to diagnose the degree of stochastization in the plasma based on a comparison of numerically modeled magnetic footprints on the divertor targets with experimental data. These results are compared to first results from $3 D$ modeling with the EMC3/EIRENE plasma and neutral transport code $[15,16]$.

\section{Dependence of ELM suppression on $q_{95}$, I-coil phase and parity}

At DIII-D the perturbing magnetic field $B_{r}^{m n}\left(\Psi_{N}\right)$ consists of three contributions [11]: components of the internal machine error field (EF), the $n=1 \mathrm{C}$-coil field applied for EF correction and the $n=3$ RMP field from the I-coils. Parity, magnitude and toroidal phase of the I-coil field as well as $q_{95}$ determine if ELM suppression is achieved, and as the ELM characteristics depend on $\bar{\delta}$ and $\nu_{e}^{*}$, the ELM suppression does too [6]. 
In figure 1 an example is shown for a dedicated scan of (a) toroidal phasing of the I-coil field, (b) $q_{95}$ as a resonance parameter for both toroidal phases $\varphi_{I C}$ and (c) parity of the I-coil field with $\varphi_{I C}=60^{\circ}$. Discharges were performed at a toroidal field of $B_{T}=-1.89 \mathrm{~T}$ and plasma current of $I_{p}=1.54 \mathrm{MA}$ with C-coils set to standard $n=1$ error field correction [11]. Figure 1(a) shows two discharges in even parity, i.e. up-down symmetric I-coil currents. The two applicable I-coil phases $\varphi_{I C}$ were switched from $\varphi_{I C}=60^{\circ}$ to $\varphi_{I C}=0^{\circ}$ and for both phases ELM suppression was achieved. Energizing the I-coils $\left(I_{I C}=4.1 \mathrm{kA}\right)$ leads to a $40 \%$ reduction in pedestal electron density $n_{e} p e d(t)$ - the so called particle pump out, characteristic for ELM suppressed RMP H-modes at DIII-D $[17,18,19]$. At the same time, pedestal electron temperature $T_{e} p e d(t)$ is reduced by $20 \%$ due to convective energy losses and the normalized ratio of plasma pressure and magnetic field, $\beta_{N}$, as well as the energy enhancement factor, $H_{98 y 2}$, decrease by $20 \%$. It is important to note that in other cases with I-coil RMP perturbation optimized for maintaining confinement properties, ELM suppression was achieved with much more moderate $\Delta n_{e}$ ped $<10 \%$ and with even increasing pedestal $T_{e}$ and $T_{i}$, and therefore maintained $H_{98 y 2}$ [6]. Plasmas presented here were operated at $q_{95}=3.52$ at $\nu_{e}^{*}=0.15$ and $\bar{\delta}=0.61$, i.e. ITER-Similar Shape plasmas at ITER relevant $\nu_{e}^{*}[6]$.

Discharges shown in figure $1(\mathrm{~b})$ document the resonant character of ELM suppression. Here, the edge safety factor $q_{95}$ was ramped down from $q_{95}=4.1$ to $q_{95}=3.15$ by ramping up the plasma current. This $q_{95}$ ramp was performed for both toroidal phases. The width of the resonance window $\Delta q_{95}$ for complete ELM suppression shrinks from $\Delta q_{95}=4.0-3.3=0.7$ for $\varphi_{I C}=60^{\circ}$ phasing [figure 1(b), grey shaded

area] to $\Delta q_{95}=3.6-3.4=0.2$ for $\varphi_{I C}=0^{\circ}$ [figure 1(b), red shaded area]. The role of $\varphi_{I C}$ in ELM suppression $([9,11])$ is also apparent in figure 1 (a) for $3.5 \mathrm{~s}<t<4.0 \mathrm{~s}$ where $I_{I C}$ is ramped down slowly. The ELMs come back already at $I_{I C}=2.8 \mathrm{kA}$ for $\varphi_{I C}=0^{\circ}$ while for $\varphi_{I C}=60^{\circ}$ they re-establish at a lower current level of $I_{I C}=1.0 \mathrm{kA}$.

Figure 1(c) depicts the dependence of ELM suppression on I-coil parity. ELM suppression was achieved for even parity at $\varphi_{I C}=60^{\circ}$ and also at higher $\nu_{e}^{*}=0.55$. However, ELM suppression gets lost when applying the I-coil field in odd parity, i.e. up-down antisymme ric I-coil currents. In this case also the particle pump out is not apparent anymore. These examples show that the actual resonance spectrum in DIII-D is determined by the external fields from I- and C-coils and the internal error fields of the machine [11]. As for ITER the internal EF of the actual machine can not be predicted, a flexible coil setup which allows for compensation of these EF effects and for generation of an useful RMP spectrum needs to be designed and implemented [9].

\section{Concept for 3D transport analysis at DIII-D}

Using the TEXTOR high field side (HFS) DED coils, resonant perturbations with base poloidal (m) and toroidal (n) mode numbers, $m / n=3 / 1,6 / 2,12 / 4$ can be generated [20]. The maximum local normalized perturbation amplitude, i.e. the ratio $\tilde{B}_{r}^{m, n}=B_{r}^{m, n} / B_{T}(q)$ of the resonant magnetic field components $B_{r}^{m, n}$ at a given $q=m / n$ 
surface to the toroidal magnetic field $B_{T}(q)$ at this surface is typically $\tilde{B}_{r}^{6,2} \sim 2 \times 10^{-2}$. This is two orders of magnitude higher than typical DIII-D values, e.g $\tilde{B}_{r}^{9,3} \sim 4 \times 10^{-4}$ leading to larger margins for exploration [21]. For TEXTOR, modeling of the stochastic magnetic field structures was performed in the vacuum paradigm with the GOURDON field line tracing code and the ATLAS simplectic mapping code [22]. Two characteristic, interwoven topological domains determine the topological and transport properties. A Laminar Zone $[23,24]$ exists with short connection length $\left(L_{c}\right)$ field lines and therefore prevailing direct parallel transport to the DED target. These field lines are clustered at TEXTOR-DED into extended magnetic flux tubes acting as a helical scrape off layer (SOL). They are embedded into a domain of Stochastic field lines $\ddagger$ with large $L_{c}$, a high field line diffusion coefficient $D_{f l} \sim 10^{-5} \mathrm{~m} / \mathrm{m}^{2}$ and therefore diffusive transport characteristics. Both domains have been identified for TEXTOR-DED in magnetic modeling [24], 2D [25] and 3D [26] transport modeling and in direct comparison to experimental results on local transport features in the perturbed edge layer $[12,13,14]$.

However, in the open chaotic system formed by DED, field lines from both domains connect directly to the DED target. The stochastic, long $L_{c}$ field lines hitting the target in the helical pattern aligned along the DED coils [27, 28] represent the imprint of a generic topological property of stochastic systems - the stable and unstable invariant manifolds of the hyperbolic fixed points of the last intact resonant island chain. For detailed explanation we refer e.g. to [29], but as this terminology is central for the further explanation we give a basic summary and a simplification for the discussion to follow. In an Hamiltonian description of the magnetic field line dynamics, the periodicity of each rational surface generates fixed points. Field lines run towards these fixed points with a periodicity matching the mode number of the perturbation. The field lines follow the invariant manifolds as they represent the topological space of the solutions of the equations of motion for the field lines. An invariant manifold is called stable when it goes positive in the hamiltonian time coordinate (here toroidal angle $\varphi$ ) and unstable when it runs negative in time towards the related fixed point. Field lines follow the stable and unstable manifolds and, in the event that any manifold hits the target, the imprint guides the field lines to the intersection point, forming a so called tangle. These tangles then form a pattern which reflects the generic stochastization of the system. In simplified words, the tangle of the stable and unstable manifolds represents a direct connection from the related resonant island chain towards the wall. Therefore identification of the tangles allows for direct diagnosis of the degree of stochastisation and of the penetration depth of the RMP field.

For TEXTOR it was shown that the measured target pattern followed directly the

$\ddagger$ In literature commonly field lines with long $L_{c}$ and decorrelated behavior are termed as ergodic field lines (e.g. [14, 22, 34, 36, 38]). However, as we will describe later, application of the Hamiltonian formalism requires generation of fixed points which prohibit ergodization of a complete radial domain in the perturbed edge layer. Only specific sub-volumes in between the invariant manifolds of the hyperbolic fixed points can be ergodized. Therefore we will use the term stochastic instead throughout the paper as the stochastic nature is given in any case for the majority of these field lines. 
magnetic footprint modeled in the vacuum approach $[30,31]$. Here the tangle of the last intact resonant island chain connected directly to the target. In measuring the tangle imprint in heat and particle fluxes, it was verified that for TEXTOR plasmas without excitation or locking of tearing modes driven by the DED field [32], the vacuum approach was in good agreement with experimentally identified target pattern. At DIII-D the divertor separatrix causes a major topological difference compared to the TEXTOR case. While at TEXTOR the helical, pitch resonant perturbation of the DED forms resonant islands and their X-points, for DIII-D plasmas in single lower null divertor configuration, one divertor $\mathrm{X}$-point is formed due to a strong external $n=0$ poloidal field. Therefore two different kinds of generic manifolds have to be distinguished for the DIII-D case $[33,34,35]$. As the divertor separatrix is very sensitive to any kind of small, non-axisymmetric perturbation [35] it splits into a set of invariant manifolds which cause the lobe structure seen in figure 2(a). Here, the shortest path from the poloidal plane to the divertor target is shown in the so called Laminar Plot representation for DIII-D discharge \#129194 at $t=3000 \mathrm{~ms}$ modeled with TRIP-3D. The manifold lobes form on the divertor target a striated pattern as depicted in figure 2(b) where the complete connection length of field lines is shown along toroidal angle $\varphi$ and the distance $s_{\text {wall }}$ along the DIII-D wall measured from the inner strike point towards the inner SOL. The outer boundary of the lobes - in the footprint pattern as well as in the laminar plot — is defined by the perturbed separatrix manifolds.

However, while the separatrix perturbation is non-resonant, the internal magnetic structure of the lobes is formed by the invariant manifolds of resonant island chains inside the plasma. The resonant field leads to the formation of magnetic islands and, for high enough perturbation, the stable and unstable manifolds of neighboring island chains cross and lead to stochastic field line diffusion [33]. Therefore, unlike TEXTOR, many rational flux surfaces from the stochastic boundary connect to the target and they form the nested, boomerang like structures seen in figure 2(b). Deeper rational surfaces show up in a boomerang of long connection length field lines at larger distance from the tip of the lobe. Identification of these boomerangs in experimental footprint pattern - as successfully executed at TEXTOR - allows one to identify directly the fragmentation of the related resonant island chain due to stochastisation. In between the resonant tangles, areas of short connection length are apparent in the magnetic footprint pattern. These short $L_{c}$ regions are comparable to the laminar flux tubes identified numerically and experimentally at TEXTOR as those field lines do not mix with the stochastic interior but connect on short distances to the divertor target. These laminar flux tubes are seen in the laminar plot of figure 2(a) as a mesh of white areas in the X-point region. From here field lines connect into the flux tube areas inside of the footprint pattern. As those flux tubes have an extension of several centimeters in the poloidal and radial direction, they can act as an effective sink towards the target from inside of formerly enclosed flux surfaces.

As the stochastic boundary modeled in the vacuum approach consists of open, perturbed field lines, the radial penetration of these open field lines on topological 
and transport lengths scales (electron mean free path $\lambda_{e}$ ) is important in analyzing the target pattern. As a topological quantity we employ the Kolmogorov Length as measure for divergence of neighboring field lines [36] and we use a value obtained with a simplectic mapping scheme for poloidal divertor tokamaks described in [34]. In figure 10 of that paper radial profiles of $L_{K}\left(\Psi_{N}\right)$ are displayed. With the parameters of discharge \#132732 the scaling of the perturbation strength with the RMP coil distance estimates $L_{K}=200 \mathrm{~m}$. For $\lambda_{e}$ one finds $\lambda_{e} \sim 10^{3} \mathrm{~m}$ with typical pedestal values of $T_{e} \sim 2 \mathrm{keV}$ and densities of $n_{e} \sim 1.0 \times 10^{19} \mathrm{~m}^{-3}$ [37]. In order to compare these lengths to the connection length value of open field lines we analyze the perturbed boundary for DIII-D discharge \#132732 in a probing field line analysis by launching 14400 field lines from outside $\Psi_{N}>0.8$. The connection length value $L_{c}$ from the point of minimal $\Psi_{N}$ reached by the field line is compared to $L_{K}=200 \mathrm{~m}$ and $\lambda_{e}$. The result is shown in figure 3 . Field lines with $L c<L_{K}$ are classified as laminar field lines following [38]. Therefore, we found that the laminar zone extends inward to $\Psi_{\text {lam }}=0.972$ and the complete stochastic edge layer in to $\Psi_{\text {stoch }}=0.816$, i.e. the laminar region as extension of the SOL has a small extend while decorrelated field lines fill the largest part of the stochastic edge. They still have $L_{c}<\lambda_{e}$ and hence direct parallel heat conduction along these open field lines is expected. However, to discuss the heat and particle fluxes along these open field lines, the location of particle and heat sources needs to be considered. As sketched in figure 3, the main particle sources are located in the SOL due to hydrogen recycling while the main heat source is deposited by beam heating inside the core plasma. Therefore particles ionized inside the laminar field lines can be drained towards the target directly while heat needs to be transported perpendicular to the field lines into the stochastic edge region. With the same topological analysis for TEXTOR [21, 39] we find for typical $\tilde{B}_{r}^{6,2} \sim 10^{-2}$, that the laminar zone extends in to $\Psi_{\text {lam }}=0.915$, about half the way to the inner boundary of the complete perturbed edge region $\Psi_{\text {stoch }}=0.815$.

In summary the topological considerations when compared to experimental and numerical TEXTOR results suggest — based on the vacuum paradigm - the approach for 3D transport analysis depicted schematically in figure 4. Figure 4(a) depicts the DIII-D perturbed magnetic boundary in poloidal angle $\vartheta$ versus normalized poloidal flux $\Psi_{N}$ as radial coordinate. Starting from the confined volume, application of RMP produces formation of resonant magnetic islands and island overlap. The island overlap is initiated by intersection of stable and unstable manifolds of neighboring resonant islands - this is the source of stochastization of the edge layer [31, 33]. Analytical $1 D$ modeling of ELM suppressed H-modes as described e.g. in [40] give a functional description of radial particle and heat transport in such an stochastic domain. In this model an enhancement of radial particle transport for $\Psi_{N}>0.96$ due to stochastic field line diffusion step size of $D_{f l}(r)=5 \times 10^{-5} \mathrm{~m}^{2} / \mathrm{m}$ explained the experimentally observed pedestal density reduction. Thermal transport modeling was performed with the E3D thermal transport code [37], showing that additional thermal transport due to stochastic field line diffusion, i.e. effectively an increase in $\chi_{\perp}$, causes collapse of the temperature profiles. A collapse of the temperature profile is not observed in the 
experiment, thus screening of the external field and/or limitation of the parallel heat flux [41] are candidates for explaining this difference. In addition to stochastic field line diffusion, remnant and intact magnetic islands are able to enhance both radial heat and particle transport even more strongly [42, 43]. This mixture of stochastic field lines and remnant islands suggests an enhancement in effective radial transport filling the laminar zone and the perturbed separatrix as visualized in figure 4(b). Both projected parallel and field line perpendicular heat and particle fluxes fill the lobes causing a striated target pattern. The laminar flux tubes are filled exclusively by radial transport as these field lines represent correlated magnetic flux tubes connecting directly to the target without penetrating into the deeper stochastic edge region inside $\Psi_{N}<0.96$ for this example. Filling of the target lobes sketched in figure 4(c) is therefore given by the diffusive radial influx and by the projected parallel fluxes along the open field lines. It is important to note that even without penetrating RMP fields and formation of a stochastic interior, the perturbed separatrix itself forms the striated footprint pattern. Therefore the lobes shall be filled by the natural anomalous plasma transport in any case and possibly also by additional parallel transport along perturbed, open field lines in the event that the stochastic edge is formed as analyzed above.

\section{Experimental pattern of perturbed magnetic field topology at DIII-D}

In this section experimental data are shown in order to reveal experimentally features of the topology described before.

\subsection{Detection of separatrix striation in target particle and heat fluxes}

Striation of the divertor target pattern during ELM suppression was detected in heat and particle fluxes as reported in plasmas for high $\nu_{e}^{*}$ [35] and it was expected from 3-D thermal transport modeling with the $E 3 D$ code also at low $\nu_{e}^{*}$ and low triangularity [37]. Here we compare directly heat and particle flux patterns for ITER similar shape, high triangularity plasmas at ITER relevant low $\nu_{e}^{*}=0.2$ with the modeled topology of the magnetic footprints. Figure 5 shows experimental evidence for striation of the divertor footprint in direct comparison to the position and length of the impinging open field lines from the stochastic interior. Data are modeled and measured in discharge \#129194 at $t=3000 \mathrm{~ms}$ during complete ELM suppression. The discharge setup was the same as shown figure 1 (a) for $60^{\circ}$ phasing (red curve). In figure 5(a) a cut of the modeled magnetic footprint pattern is shown in the vicinity of the camera observations used and the actual observation volume is shown as rectangular box with dashed black lines. In figure $5(\mathrm{~b})$ a $D_{\alpha}\left(s_{\text {wall }}\right)$ intensity profile, taken as proportional to the incoming particle flux, is shown in comparison to the magnetic field lines hitting the target. A clear striation in particle flux is seen at this toroidal position $\varphi=145^{\circ}$ and here, three lobes are detected in magnetic modeling, too. The narrow lobes at $0.5 \mathrm{~cm}<s_{\text {wall }}<2.0 \mathrm{~cm}$ in modeling are not resolved in the measurement but they fall together in the first, larger 
$D_{\alpha}$ peak. However, the measured location of the distinct lobes is in fair agreement with the modeled lobe location. This comparison proves that the generic striation formed by the perturbed separatrix is apparent.

Figure 5(c) shows the comparison of radial heat and particle flux profiles on the inner divertor. The heat flux $q_{\perp}$ data shown were obtained with a fast framing IR camera [27] observing with a vertical line of sight in radial direction the complete inner and outer divertor areas and a fraction of $\Delta \varphi=48^{\circ}$ in toroidal direction centered at $\varphi=165^{\circ}$. An overlapping toroidal region of $\Delta \varphi=30^{\circ}$ centered at $\varphi=150^{\circ}$ is observed simultaneously using a CCD camera with a set of filters suitable to get spectral lines strongly related to the incoming particle flux $\Gamma_{\perp}$. In heat flux only the innermost of the separatrix lobes forms a large local heat flux maximum. The second lobe, detected well in particle flux, is hidden in the shoulder of the main heat flux peak, and the third lobe carries a heat flux of $q_{\perp}=0.4 \mathrm{MWm}^{-2}$. This experimental observation seems to be counter intuitive as large parallel heat conduction along all the open field lines - which penetrate as deep as $\psi_{N}=0.85$ (figure 3) — was expected on the first glance. To compare this value on a basic level to expected heat fluxes in the SOL and also parallel heat conductivities, we extract here two extremal values for such quantities.

As minimal value which shall arrive evenly in all lobes touching the target, one considers the radial heat flux caused by the perpendicular heat transport $q_{\perp S O L}=$ $P_{t o t} / A_{S O L}$ crossing the separatrix region into the SOL also in the unperturbed case. With the total power injected $P_{t o t}=8.0 \mathrm{MW}$ and the area of a flux surface at the separatrix $A_{S O L}=44 \mathrm{~m}^{2}$ we find $q_{\perp S O L}=0.18 \mathrm{MWm}^{-2}$. This is the anomalous radial heat transport which determines the energy confinement time $\tau_{E}$ and therefore the radial heat flux crossing the SOL and touching the target due to the perturbed separatrix lobes even without any open field lines, as suggested in the sketch of figure 4 (b). The value of $q_{\perp}=0.4 M W m^{-2}$ inferred by IR measurement is a factor of 2 higher showing that additional parallel conduction must exist. Possible channels are the SOL parallel heat flux which passes through the lobes in the perturbed separatrix region and might be projected towards the target by the lobes. However, this would lead to a even imprint of all lobes on the target which is not seen in the measurement. Therefore parallel heat conduction along open field lines from different radial location in the perturbed boundary is needed to explain the highly located heat flux in particular around $s_{\text {wall }} \sim 0 \mathrm{~cm}$ compared to the small value in the third, outer lobe. This, however requires penetration of the RMP field to a certain radial extend. In this case, field lines with $L_{c} \sim \lambda_{e}$ connect according to the analysis presented in figure 3 , from as deep as $\Psi_{N}>0.85$ into the lobes establishing additional heat flux by parallel heat conduction $q_{\|}$.

For $q_{\|}$we evaluate as a maximal value the direct heat conduction from representative flux surface which had become stochastic according to the vacuum modeled topology. They are expected to be very high given the $T_{e} \sim 1-2 \mathrm{keV}$ and $n_{e} \sim 2-410^{19} \mathrm{~m}^{-3}$ values at $\Psi_{N}=0.85$. For the pedestal profiles of discharge $\# 129194$ at $t=3000 \mathrm{~ms}$ we 
calculated heat fluxes $q_{\|}\left(\Psi_{N_{i}}\right)$ as free streaming heat flux in the low collisionality limit $\left.q_{F S}\left(\Psi_{N_{i}}\right)=n_{e}\left(\Psi_{N_{i}}\right) T_{e}\left(\Psi_{N_{i}}\right)^{3 / 2} / \sqrt{(} m_{e}\right)$ discussed in [41]. In addition we used the heat flux limitation applied in that paper and calculated $q_{F S \text { lim }}=\xi \times q_{F S}$ with two values for $\xi=0.03,0.1$ following [41]. We assume for this description that, by large amounts of open field lines, parallel heat conduction inside the plasma is radially displaced and directly channeled to the target plates. Table I shows the results for four different $\Psi_{N_{i}}$ values. Restricting the analysis to the values from Ref. [41], the only value in the table that is the same order of magnitude as the $q_{\|}<12 \mathrm{MWm}^{-2}$ measured in the third, outer lobe is for the case of direct parallel conduction at $\Psi_{N}=0.99$ with heat flux limit of $\xi=0.03$. However, it is important to note that the values obtained are limited and reduced in the actual perturbed plasma by several facts. First the field lines connect into both directions and only a small fraction of the perturbed field lines leave the plasma on $L_{c} \sim \lambda_{e}$ from radial regions $\Psi_{N}<0.95$ as depicted in figure 3 and discussed in [10]. This shall reduce the effective heat flux density by up to order of magnitude. Last but not least, no local balance of the incoming radial heat flux against these parallel heat fluxes was included. The parallel out flux must be maintained by a radial influx into the actual heat sink region caused by the open field lines. Due to the particle pump out, the density drops by a factor of two in these shots which leads to a reduction of neoclassical radial heat conductivity $\chi_{\perp} \sim n^{2}$ available to the local heat sink region by a factor of 4 . Therefore the complex $3 D$ pathway of the open, perturbed field lines need to be considered for a good estimate of the actual parallel heat fluxes. The complete analysis to understand the transport processes filling the target lobes needs $3 D$ plasma and neutral transport modeling. First results will be presented in the last section of this paper.

However, the dynamics of the open field lines invented by vacuum modeling strongly suggests an explanation for the pronounced maximum of heat flux in the vicinity of the formerly unperturbed separatrix. As described in [33], most of the stochastic field lines, following the invariant manifolds of the resonant island chains, intersect the target first inside the innermost lobe, entering the plasma again and touching the target consecutively again in the second and third outer lobes. In addition figure 2(b) shows that the resonant tangle layers from deeper flux surfaces are stretched out into a very thin structure around $0 \mathrm{~cm}<s_{\text {wall }}<2.5 \mathrm{~cm}$. Due to this two topological reasons, most heat flux is carried to this location where the pronounced and wide heat flux maximum is measured. The two adjacent lobes located here can not be resolved by the IR measurement due to draining of the incoming fluxes by perpendicular heat transport in between the lobes and into the adjacent SOL. Therefore, the new radial width of this heat deposition profile is determined by the perturbed separatrix topology, i.e. the stretched layer of separatrix lobes which replaces the formerly unperturbed strike line. Its width increases with applied perturbation, leading to a decrease of the peaking factor $\Delta q_{\perp}=\max \left[q_{\perp}\left(s_{\text {wall }}\right)\right] \times \int d s_{\text {wall }} / \int q_{\perp}\left(s_{\text {wall }}\right) d s_{\text {wall }}$. For the inter ELM phase high peaking of $\Delta q_{\perp}=10.4$ is observed while it is stabilized during the ELM suppressed 
phase at a low value of $\Delta q_{\perp}=3.6$. In contrast, the interior of the outermost (third) lobe is determined by flux surfaces radially closer to the separatrix as it incorporates in the observation volume the boomerangs of the tangles from five resonant surfaces from $\psi_{N}>0.97$. Therefore the heat flux measured in this outer lobe compares good to the value previously estimated for parallel heat conduction along the open field lines with $\xi=0.03$ from the outer edge region. These findings give substantial evidence that during RMP application the deposition of heat is correlated with the topology of the elongated resonant surfaces and that parallel heat conduction along open, perturbed field lines from some deeper interior must exist to explain the localized heat flux maximum and the new 3-D strike line structure and width.

\subsection{Rotation of the separatrix lobes with toroidal phase of the I-coil field}

In this section we demonstrate that the striated separatrix obeys the phase $\varphi_{I C}$ of the external $n=3 \mathrm{RMP}$ field generated by the I-coils. In this experiment $\varphi_{I C}$ was changed from $\varphi_{I C}=60^{\circ}$ for discharge $\# 132731$ to $\varphi_{I C}=0^{\circ}$ for discharge \#132732. As described in section 1 both discharges showed ELM suppression. With ELM suppression, striation of the inner divertor strike point (ISP) appears as seen in CII intensity depicted in figure $6(\mathrm{~b})$ for both toroidal phases (black curve for $60^{\circ}$ phase and red curve for $0^{\circ}$ phase). We use $C I I$ here as a representative measurement for the incoming ion flux based on dominating physical sputtering in the attached inner divertor leg during ELM suppression [45]. Comparison of these averaged $I_{C I I}\left(s_{\text {wall }}\right)$ profiles (in between $2600<t<2750 \mathrm{~ms}$ ) shows that the peaks move and that the relative intensity changes.

Comparing to figure 6(a) confirms that this movement is in agreement with the $60^{\circ}$ movement in the modeled lobe position. The third stripe from the ISP $\left(S_{\text {wall }} \sim 1.8 \mathrm{~cm}\right.$ in the curve a view moves outwards as does the corresponding maxima in the profile. It is important to note that this movement shifts into the diagnostic view a region of the lobe interior that connects to one island chain deeper than the previous one. The relative change in the maxima can therefore be attributed to a correlated larger ion flux to this target location. The opposite effect is apparent in the outermost lobes around $s_{\text {wall }} \sim 4.5 \mathrm{~cm}$. Here the intensity is reduced in shifting $\varphi_{I C}$ from $60^{\circ}$ to $0^{\circ}$ because this shifts a lobe part connecting to deeper resonances away, and moves a lobe tip dominated by laminar flux tubes from $\Psi_{N}>0.97$ into the observation volume. This measurement shows that the perturbed structure is controlled by the external RMP field and in addition evidence is found for a correlation of the CII intensities with the open field lines from different resonance layers.

\subsection{Manipulation of separatrix lobes by edge safety factor $q_{95}$}

It was shown at DIII-D that ELM suppression is a resonant phenomenon and complete suppression is achieved in a quite narrow window for $q_{95}$ with a width of typically $\Delta q_{95}=0.1-0.4$ depending on $\nu_{e}^{*}$, parity and the actual RMP spectrum $[6,11]$. Here we analyze discharge \#132741 with $q_{95}$ ramp down. For this discharge ELM suppression 
was achieved practically from $3.2<q_{95}<3.9$ interrupted for $3.63<q_{95}<3.7$ by three large ELM events and some compound ELM activity. Nevertheless, this wide extension of the resonant $q_{95}$ window allows for good diagnosis of footprint structures. During this $q_{95}$ ramp the infinitesimally close rational surfaces close to the separatrix interact and rotate the separatrix tangles along the target. This characteristic is seen in heat and particle flux as shown in figure 7. Figure $7(\mathrm{~b})$ shows the $C I I$ intensity at the inner strike point (ISP) versus $q_{95}$ while in figure $7(\mathrm{c})$ the corresponding heat flux $q_{\perp}\left(s_{\text {wall }}, q_{95}\right)$ is depicted. With increasing $q_{95}$ the resonant window for complete ELM suppression is entered, and a striated pattern is apparent consisting of two narrow and one more remote stripe. During the $q_{95}$ ramp these lobes rotate through the observation volume of the cameras and new lobes depart from the unperturbed strike point position consistent with results from TRIP-3D modeling as shown in figure 5(a). Figure 7(a) shows the calculated positions of the lobes appearing in the observation volume as $q_{95}$ is increased. The lobes modeled in the vacuum paradigm follow in good agreement the measured ones. This, in combination with the results from the phase scan, provides substantial evidence that the $n=3$ I-coil field controls the position and the distance of the separatrix lobes. However, there is a dramatic difference between heat and particle flux, here measured as CII intensity, in the intensity weighting of the lobes relative to the ISP location. During ELM suppression the heat flux is about three times higher at the former ISP location $\left(s_{\text {wall }} \sim 0 \mathrm{~cm}\right)$ than in the outer lobes. In contrast the highest ion fluxes, here CII intensity, are always measured in the outermost lobe while the value around $s_{\text {wall }} \sim 0$ is nearly constant and increase as soon as a lobe with dominant contributions of laminar field lines arrives at this location. For this discussion it is important to mention, that the $C I I$ intensity is $T_{e}$ sensitive due to a pronounced maximum in the cross-section for the given density regime around $T_{e} 5-8 \mathrm{eV}$ [45]. Therefore, the relative intensity change might be matter of temperature modulation along the incoming field lines in addition to changes in the ion fluxes. Quantitative analysis of the particle fluxes from the spectroscopic $D_{\alpha}$ and $C I I$ data need more experimental work.

Nevertheless, the important difference in the heat vs. particle flux ratios in the third, outer lobes vs. the inner lobe bundle around $s_{\text {wall }} \sim 0 \mathrm{~cm}$ finds its rational in the particle vs. heat source location as discussed based on figure 3. As the heat source sits inside the plasma, heat needs to be transported radially into the region of open field lines and is dumped near $s_{\text {wall }}=0 \mathrm{~cm}$ as the largest ratio of open field lines from the deep interior intersect here. In contrast, neutrals recycling into the SOL can be drained along the open, laminar field lines from the laminar region (see figure 3) as soon as they are ionized in the outer lobe which carries obviously enough energy to do so. Therefore, the appearance of the outer lobes in particle flux and the pronounced local maximum around $s_{\text {wall }}=0 \mathrm{~cm}$ in heat flux - seen during $q_{95}$ scan $(C I I$ intensity) as well as in the case of fixed $q_{95}\left(D_{\alpha}\right.$ intensity) shown in figure 5 - can be explained by the opposite particle and heat source locations in combination with open, stochastic field lines at least in the outer vicinity of $\Psi_{N} \gtrsim 0.95$. The following results from 3-D modeling will support this approach for explanation of the observations. 


\section{Initial results from 3D modeling with EMC3/EIRENE}

The plasma and neutral transport code EMC3/EIRENE [16, 46] is used for a wide range of applications focusing on 3D fluid modeling in various non-axisymetric magnetic topologies. It is used in stellarator symmetry and helical devices [47]. It was adapted to the TEXTOR-DED geometry [48] and there validated against experiment [14] which implied code improvement, e.g. implementation of heat flux limitations [49]. Recently comparison of modeling results for limiter plasmas at TEXTOR and DIII-D was carried out showing a direct comparability of the footprint pattern at both experiments [15]. In particular it was shown for TEXTOR [15] that diagnosis of the imprint of the open resonant tangles on the inner wall depends on sufficiently low radial diffusion $D_{\perp}$ and $\chi_{\perp}$, an important guideline for the results presented below. In order to study $3 D$ transport features on TEXTOR and DIII-D with EMC3/EIRENE, the code was adapted to the poloidal divertor geometry. A detailed report on this will be published soon in a separate paper. Here first results from initial modeling are shown focusing in particular on the filling of the separatrix lobes and dependence of footprint striation on the radial transport.

Figure 8 shows electron density $n_{e}(R, Z)$ and electron temperature $T_{e}(R, Z)$ distributions modeled for discharge \#122302 with radially constant anomalous diffusion coefficients $D_{\perp}=0.25 \mathrm{~m}^{2} \mathrm{~s}^{-1}$ for particle and $\chi_{\perp}=0.75 \mathrm{~m}^{2} \mathrm{~s}^{-1}$ for conductive heat transport. Further boundary conditions were the density and temperature scrape off lengths $\lambda_{n}=30 \mathrm{~mm}$ and $\lambda_{T}=50 \mathrm{~mm}$ and the heating power injected $P_{H}=600 \mathrm{~kW}$ and density at the inner boundary of $n_{e}=2.0 \times 10^{19} \mathrm{~m}^{-3}$. This set of input parameters was chosen for test purposes and direct comparative modeling is in progress. In figure 8 it is shown that the plasma boundary reflects the non-axisymmetric features of the perturbed magnetic topology. In the main plasma region the shape of the invariant separatrix manifolds [33], determined the wiggled $n_{e}$ and $T_{e}$ shape at transition to the SOL. They act as an envelope and represent a new plasma boundary. In particular a radial filling of the lobes in the vicinity of the $\mathrm{X}$-point is apparent. Here the separatrix lobes guide - for the transport level specified - the particle and heat fluxes to the target.

This is studied in more detail by analyzing the target heat $\left(q_{\|}\right)$and particle $\left(\Gamma_{\|}\right)$ fluxes for a set of three transport coefficients. Modeling was done with $D_{\perp}=1.0 \mathrm{~m}^{2} \mathrm{~s}^{-1}$, shown in the left column of figure $9, D_{\perp}=0.25 \mathrm{~m}^{2} \mathrm{~s}^{-1}$ in the middle column and $D_{\perp}=0.10 \mathrm{~m}^{2} \mathrm{~s}^{-1}$, right column. For all modeling runs $\chi_{\perp} / D_{\perp}=3$ was kept constant. This scan clearly reveals the conclusion drawn during discussion of the experimental pattern. High radial diffusive transport yields a smear out of the imprint of the separatrix lobes in the striated target pattern. Reduction of radial diffusion concentrates the fluxes along the open field lines into the separate stripes of the magnetic topology. Given that we see all three striations of the $n=3$ footprint pattern in the measured particle flux pattern, $D_{\perp}=0.25 \mathrm{~m}^{2} \mathrm{~s}^{-1}$ and accordingly $\chi_{\perp}=0.75 \mathrm{~m}^{2} \mathrm{~s}^{-1}$ appear to be good values for this modeling. In the future this kind of comparison 
and validation against radial profiles shall allow the determination of effective radial transport coefficients in the $3 D$ perturbed topology.

However, one striking result was not discussed so far. The modeled heat and particle fluxes both show striation and obviously parallel heat conduction leads to a filling of the separatrix lobes. This is in contrast to the experiment in which neither a widening of the heat flux profile due to enhanced radial heat conduction appears nor the splitting on an equal level as seen in the experimental particle flux. This is of particular interest as the modeling grid for EMC3/EIRENE is generated in vacuum approach, too. Therefore the field line dynamics as explained before is embedded into this modeling. As the relative heat fluxes in the outer and inner lobes are similar in modeling and by a factor of 5 different in experiment additional processes need to be considered. As collisionality along the field lines connecting from the outer and inner boundary can be different, limitation of parallel heat conduction, so far described in Spitzer-Härm collisional limit [41] in the code, is a candidate. Future analysis will include the heat flux limitation implemented to EMC3/EIRENE [49] to study if this can lead to the experimentally observed ratios of heat flux in the different lobes.

\section{Discussion and Conclusion}

The experimental and numerical results presented in this paper revealed a $3 D$ perturbed magnetic topology in the RMP ELM suppressed boundary for DIII-D which shows comparable features to TEXTOR-DED. At TEXTOR the direct connection of fragmented resonant island chains to the target due to the tangles of the invariant manifolds of the hyperbolic fixed points of this island chain was detected by comparison of experimental data with magnetic field topology in vacuum approach. This showed for the TEXTOR case that this approach describes the perturbed magnetic field structure satisfactory until tearing modes are driven by the external RMP field. Up to this threshold the field was seen to penetrate linearly without field screening or field amplification. At DIII-D detection of the perturbed separatrix imprint in measured target particle and heat fluxes provides substantial evidence for 3-D perturbation of the plasma boundary. The invariant manifolds of the separatrix represent the new plasma boundary and therefore a 3-D topology exists. Striations in the measured target pattern are in agreement with magnetic modeling and it was shown that the external RMP field leads to control of target particle and heat flux into the separatrix lobes.

However, the location of deposition is contrary for particle and heat fluxes. As heat sources are located inside the core plasma, heat flows along the open field lines and is dumped into the stretched lobes close to the formerly unperturbed strike line as here the largest fraction of field lines from the deeper interior intersects the target. In contrast the dominant particle source is hydrogen recycling and therefore particle flux prevails in the outer lobes consisting of field lines from the outer plasma boundary, i.e. laminar field lines with short connection length. They drain the ionized particles into the outer lobes and accordingly the striations caused by the 3-D topology are best visible in the particle 
flux. However, this explanation is only possible if field penetration and the consecutive stochastisation as suggested in the vacuum approach with an minimal radial extend of $\Psi_{N} \approx 0.95$ takes place. This is in particular suggested by initial EMC3/EIRENE plasma and neutral transport modeling. Here, striation in qualitative agreement with the experimental observation was seen only for low perpendicular particle and heat diffusion coefficients $D_{\perp}=0.25 \mathrm{~m}^{2} \mathrm{~s}^{-1}$ and $\chi_{\perp}=0.75 \mathrm{~m}^{2} \mathrm{~s}^{-1}$. For higher perpendicular transport the striated pattern was smeared out leading to a blurred strike point pattern. Therefore the appearance of the narrow striated target pattern in both, heat and particle flux as observed in experiment requires dominant transport along open field lines. The parallel heat conduction must come from a region deeper than the extend of the laminar zone $\left(\Psi_{N}=0.97\right)$ as the location of the particle and heat flux maxima is determined by field lines from the particle source region vs. field lines from the deeper interior.

It is important to note that the results described here are on the present data set consistent also with the perturbed magnetic topology modeled including different screening mechanisms $[8,51,53,54,55]$. Here, toroidal rotation, neoclassical viscous forces and excitation of MHD modes lead to a plasma feedback and in all cases to a screening of the RMP field amplitude on deeper resonant layers $\left(\Psi_{N} \lesssim 0.95\right)$. However, in the edge region $\Psi_{N} \gtrsim 0.95$ this analysis shows that strong island overlap and effective stochastisation still takes place. Explanation of the results presented in this paper requires open field lines from the laminar region and somewhat deeper, which suggests a necessary radial extent of $\psi_{N} \approx 0.95$. Exact specification of the extent of these zones in the perturbed edge layer by application of screening models and/or field amplification is strongly motivated by these experimental results and will be presented in future work. However, as this analysis was largely motivated from TEXTOR results and initial attempt was made modeling screening coefficients due to rotational screening in a visco-resistive drift-MHD model as described in references [50, 51]. This allows to compare the plasmas on both experiments discussed in this paper based on the field penetration characteristics expected from this approach. We use the pedestal input profiles and rotation profiles from DIII-D discharge \#129194 and for TEXTOR parameters for typical DED $m / n=6 / 2$ discharges (see [52]). Values on the $q=3$ surface were analyzed, i.e. screening coefficients for the $m=6, n=2$ components at TEXTOR and $m=9, n=3$ components at DIII-D were deduced. At DIII-D the expected RMP amplitude damping due to rotational screening $f_{s}=0.8$ is a factor of four larger than for TEXTOR $\left(f_{s}=0.2\right)$ at $\Psi_{N}=0.93$, where $q=3$ is located. Here large magnetic shear actually drives edge stochastisation effectively and formation of the stochastic edge layer is in particular in this region very sensitive to screening of the RMP field. This shows again that plasma feedback needs to be included in the analysis of the magnetic topology and in the EMC3/EIRENE transport modeling for a complete discussion of the experimental data presented here and a possible conclusion on the radial extend of the stochastic edge region as described in the vacuum approach. 
On basis of the material presented, the 3-D topology shown in experiment with at least a narrow stochastic layer in $\Psi_{N} \gtrsim 0.95$ is suggested as an ideal candidate to interact with further transport processes beyond the diffusive and convective radial as well as the direct parallel transport picture employed in this paper. The invariant separatrix manifolds span a new 3-D boundary for the plasma and penetrating field lines interact with the radial profiles of the plasma directly changing the particle source distribution. They shall interact also with the plasma confinement and radial profiles by manipulation of e.g. electrical field properties due to the open, perturbed field lines. Resolution of profile effects and link to turbulence properties, neoclassical effects and

effects of the 3-D equilibrium are possible on the basis of the numerical and experimental results presented in this paper.

\section{Acknowledgments}

This work was supported by the US Department of Energy under DE-FC0204ER54698, DE-FG02-07ER54917, DE-AC52-07NA27344, DE-AC05-06OR23100, DEAC04-94AL85000, and by German Research Foundation under UN 265/1-1 and SP $265 / 1-1$. 


\section{References}

[1] Huysmans G T A et al. 2005 Plasma Phys. Control. Fusion 47 B165

[2] Loarte A et al 2003 Plasma Phys Control Fusion 451594

[3] Fenstermacher M E et al 2003 Plasma Phys Control Fusion 451597

[4] Fundamenski W et al 2008 J Nucl Mater (submitted)

[5] Evans T E et al 2006 Nature Physics 2419

[6] Evans T E et al 2008 Nucl Fusion 48024002

[7] Liang Y et al 2007 Phys Rev Lett 98265004

[8] Becoulet M et al 2007 Nucl Fusion 48024003

[9] Schaffer M et al 2008 Nucl Fusion 48024004

[10] Evans T E et al 2002 Phys Plasmas 94957

[11] Fenstermacher M E et al 2008 Phys Plasmas 15056122

[12] Jakubowski M et al 2006 Phys Rev Lett 96035004

[13] Lehnen et al 2005 Plasma Phys Control Fusion 47 B237

[14] Schmitz O et al 2008 Nucl Fusion 48024009

[15] Frerichs H et al 2008 Europhysics Conference Proceedings, EPS (to be submitted)

[16] Feng Y et al 2004 Contrib Plasma Phys 4425

[17] Evans T E et al 2004 Phys Rev Lett 92235003

[18] Moyer R et al 2008 J Nucl Mater (submitted)

[19] Unterberg E A et al 2008 J Nucl Mater (submitted)

[20] Finken K H et al 1999 Nucl Fusion 39637

[21] Schmitz O et al 2008 J Nucl Mater (submitted)

[22] Abdullaev S S et al 2005 "The Structure of Magnetic Field in the TEXTOR-DED” vol 45 of Energy Technology (Schriften des Forschungszentrums Juelich)

[23] Nguyenn F et al 1997 Nucl Fusion 37742

[24] Abdullaev S S et al 2003 Nucl Fusio 43299

[25] Eich T et al 2000 Nucl Fusion 401757

[26] Kobayashi M et al 2004 Nucl Fusion 44 S64

[27] Jakubowski M W 2004 Nucl Fusion 44 S1

[28] Schmitz O et al 2007 J Nucl Mater 363-365 680

[29] Evans T E 2008 "Implications of Topological Complexity and Hamiltonian Chaos in the Edge Magnetic Field of Toroidal Fusion Plasmas," Chaos, Complexity and Transport: Theory and Applications Ed. C, Chandre, X. Leoncini, and G. Zaslavsky (Singapore: World Scientific Press)

[30] Jakubowski M W et al 2007 J Nucl Mater 363371

[31] Wingen A et al. 2007 Phys Plasmas 14042502

[32] Koslowski H R et al 2006 Plasma Phys Control Fusion 48 B53

[33] Wingen A et al 2008 Phys Plasmas (submitted)

[34] Abdullaev S S et al 2008 Phys Plasmas 15042508

[35] Evans T E et al 2005 Journal of Physics: Conference Series 7174

[36] Ghendrih Ph et al 1996 Plasma Phys Control Fusion 381653

[37] Joseph I et al 2008 Nucl Fusion 48045009

[38] Ghendrih $\mathrm{Ph}$ et al 2002 Nucl Fusion 421221

[39] Jakubowski M W et al 2007 Plasma Phys Control Fusion 49, S109-S121

[40] Tokar M et al 2008 Phys Plasmas, in press

[41] Tokar M et al 2007 Phys Rev Lett 98095001

[42] Spakman G W et al 2008 Nucl Fusion (submitted)

[43] Gupta A et al 2008 Phys Plasmas 15034503

[44] Jakubbowski M et al 2008 J Nucl Mater (submitted)

[45] Isler R C et al 2007 Phys Plasmas 14012506

[46] Reiter D et al 2005 Fusion Sci Technol 47172 
[47] Feng Y et al 2008 Nucl Fusion 480214012

[48] Kobayashi M et al 2004 Nucl Fusion 44 S64

[49] Harting DD et al 2008 Contrib Plasma Phys 481

[50] Tokar M et al 2006 Phys Rev Lett 13032503

[51] Nicolai A et al 2008 Nucl Fusion 024008

[52] Lehnen M et al 2008 Plasma and Fusion Research (accepted for publication)

[53] Heyn M.F. et al 2008 Nucl Fusion 024005

[54] Yu Q. et al 2008 Nucl Fusion 024007

[55] Menard J.M. et al, 2008, this conference, submitted to Plasma Phys Control Fusion 
Table I. Parallel conductive heat fluxes along open field lines.

\begin{tabular}{lllll}
\hline & $\Psi_{N}=0.85$ & $\Psi_{N}=0.90$ & $\Psi_{N}=0.95$ & $\Psi_{N}=0.99$ \\
\hline$q_{F S}\left(M W m^{-2}\right)$ & $90 \times 10^{3}$ & $57.6 \times 10^{3}$ & $19.14 \times 10^{3}$ & $1.14 \times 10^{3}$ \\
$q_{F S} \times 0.1\left(M W m^{-2}\right)$ & $9.4 \times 10^{3}$ & $5.76 \times 10^{3}$ & $1.9 \times 10^{3}$ & 114 \\
$q_{F S} \times 0.03\left(M W m^{-2}\right)$ & $2.8 \times 10^{3}$ & $1.7 \times 10^{3}$ & 575 & 35 \\
\hline
\end{tabular}




\section{List of Figure Captions}

Fig. 1. Complete ELM suppression for variation of toroidal phase [(a) DIII-D discharge \#132731 $\left(60^{\circ}\right)$ and \#132732(0 $\left.\left(0^{\circ}\right)\right]$ and dependence on $q_{95}$, [(b) DIII-D discharge \#132741 $\left(60^{\circ}\right)$ and \#132742( $\left(0^{\circ}\right)$ ], and parity [(c) DIII-D discharge \#132748(even) and $\# 132749$ (odd) $]$ of the $n=3$ RMP I-coil field. Plots show from top to bottom $q_{95}(t), D_{\alpha}$ intensity at the inner divertor, the I-coil current applied, $n_{e}(t)$ and $T_{e}(t)$ on top of the H-mode pedestal.

Fig. 2. (a) Perturbed separatrix topology as distribution $L_{c}(R, Z)$ of shortest connection length in $m$, (b) Magnetic footprint pattern at the inner strike point position [complete $\left.L_{c}\left(s_{\text {wall }}, \varphi\right)\right]$ for DIII-D discharge \#129194 at $t=3000 \mathrm{~ms}$. Note that the discontinuity at $s_{\text {wall }}=6 \mathrm{~cm}$ is due to the boundary between the $45^{\circ}$ tile surface and the centerpost tile surface.

Fig. 3. Penetration of open, perturbed field lines into the DIII-D stochastic edge.

Fig. 4. Schematic view on transport in the perturbed $3 D$ boundary at DIII-D. (a) An unfolded view of the topology with sketched confined volume, remnant resonant islands, the stochastic field lines and the unperturbed (red dashed line) and perturbed separatrix line with the separatrix lobes and the laminar zone as SO). (b) The X-point region with the separatrix lobes. The inner (ISP) and outer strike point (OSP) and the SOL and private flux region (PFR) are marked. (c) The intersection of the lobes with the divertor target including the lobes of the resonant island chains and heat and particle transport channels filling the separatrix lobes.

Fig. 5. Identification of the perturbed separatrix striations on the inner divertor footprint pattern. (a) A cut of the magnetic footprint $L_{c}\left(\varphi, s_{\text {wall }}\right)$, (b) A direct comparison of particle flux profile $\Gamma_{\perp}\left(s_{\text {wall }}\right)$ (measured as $D_{\alpha}$ intensity) with $L_{c}\left(s_{\text {wall }}\right)$. (c) Depicts a direct comparison of the measured $\Gamma_{\perp}\left(s_{\text {wall }}\right)$ and heat flux $q_{\perp}\left(s_{\text {wall }}\right)$ profiles at the same toroidal angle.

Fig. 6. Control of lobe position by toroidal phasing of the I-coil field. Please note that the CII profiles are dashed in the region where the signal to noise ratio is small.

Fig. 7. Rotation of the separatrix lobes in heat (c) and particle flux (b) data during $q_{95}$ ramp down, plot (a) shows the rotating lobes as modeled with TRIP-3D.

Fig. 8. Results from initial EMC3/EIRENE modeling: (a) electron density and (b) temperature distribution for RMP discharge $\# 123302$ for $t=3000 \mathrm{~ms}$ at $\varphi=0^{\circ}$.

Fig. 9. Results from initial EMC3/EIRENE modeling: heat and particle flux at inner strike point for RMP discharge \#123302 for 1/3 of the toroidal circumference, i.e. one symmetry section of the $n=3$ I-coil field (C-coil field applied for EFC is not used in 
the present modeling). Input transport coefficients were varied form $D_{\perp}=1.0 \mathrm{~m}^{2} \mathrm{~s}^{-1}$ to $D_{\perp}=0.25 \mathrm{~m}^{2} \mathrm{~s}^{-1}$ and $D_{\perp}=0.1 \mathrm{~m}^{2} \mathrm{~s}^{-1}$ while $\chi_{\perp} / D_{\perp}=3$. The dark vertical lines are boundaries of the toroidal grid cells in the model while the white line marks the location of the diagnostic in the modeling results. 
(a) even parity, $60^{\circ}$ phase $\mid$ even parity, $0^{\circ}$ phase
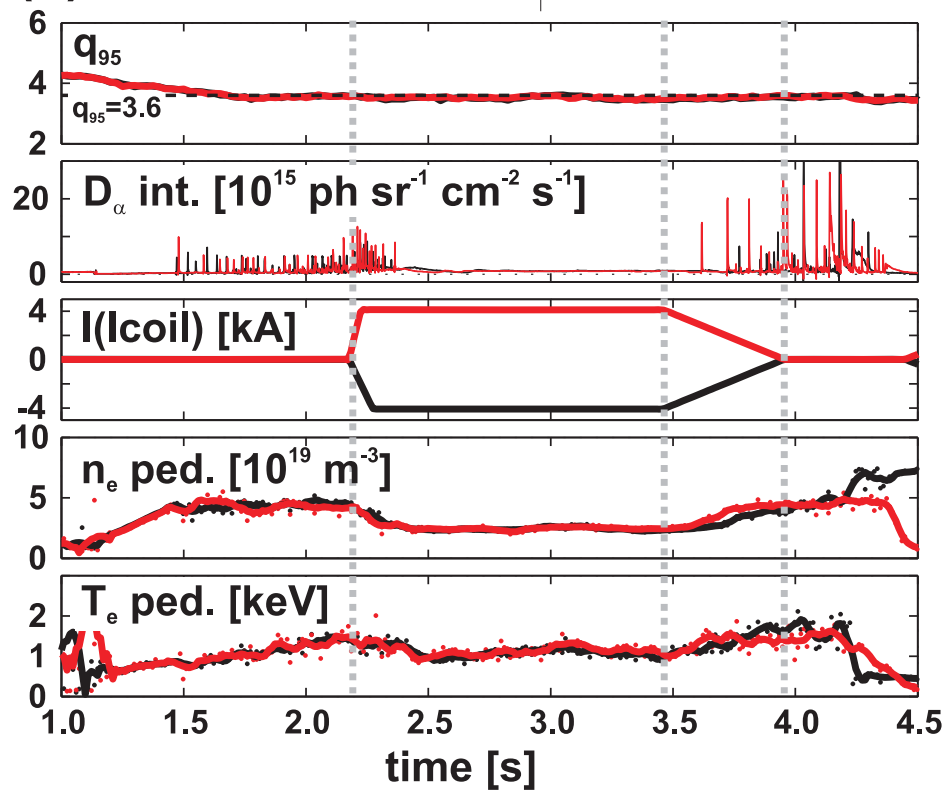

(b) even parity, $60^{\circ}$ phase $\mid$ even parity, $0^{\circ}$ phase

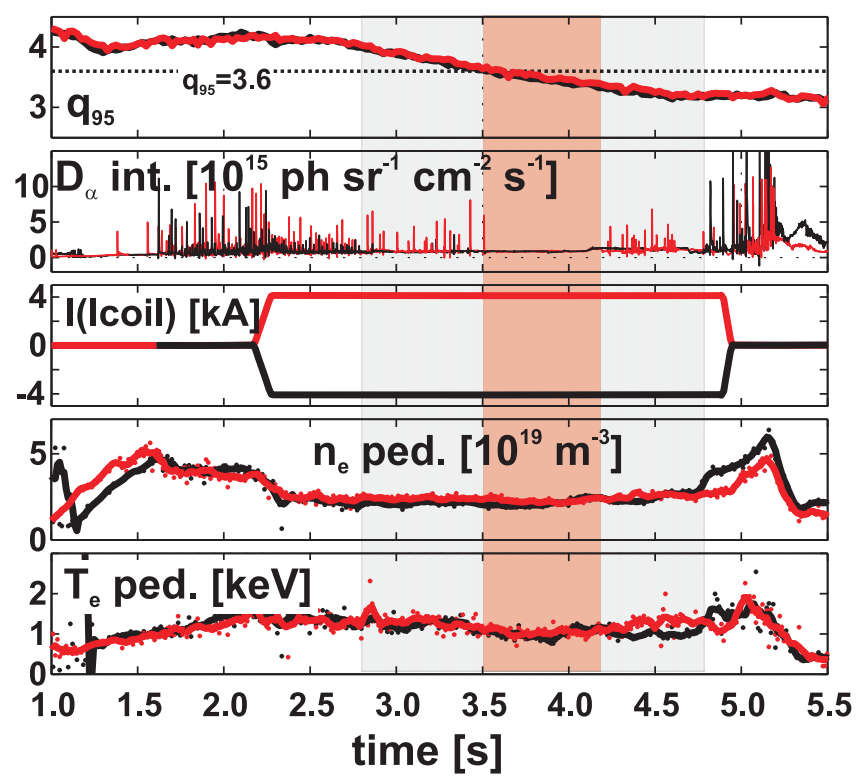

(C) odd parity, $60^{\circ}$ phase $\mid$ even parity, $60^{\circ}$ phase

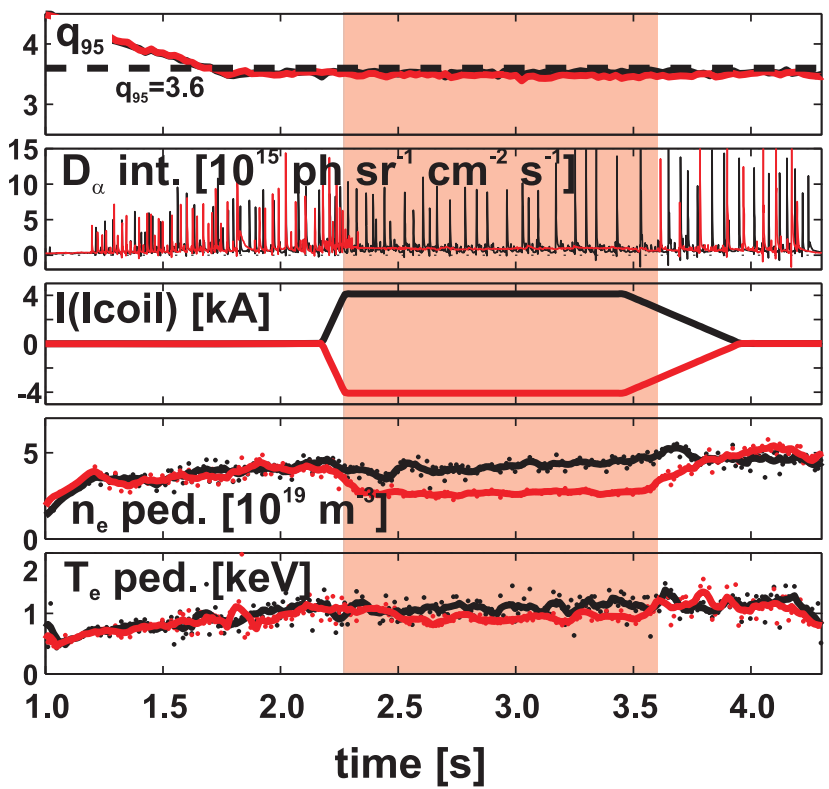



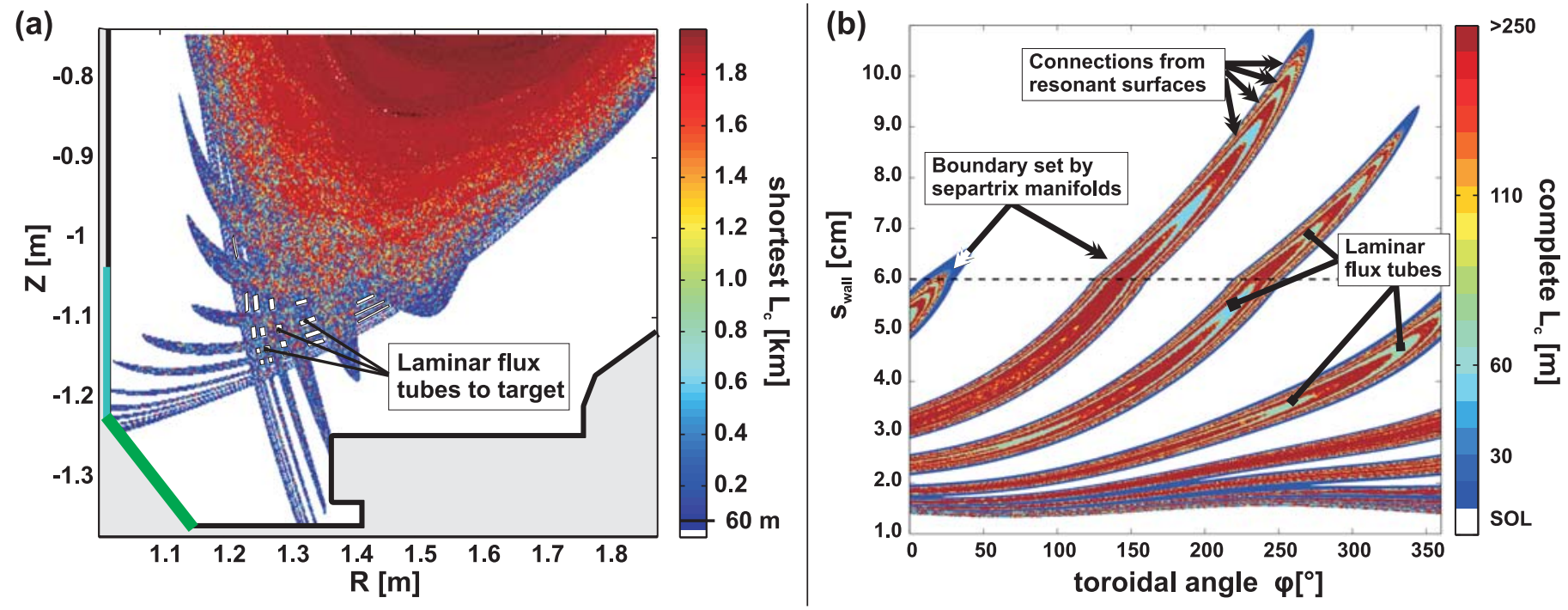


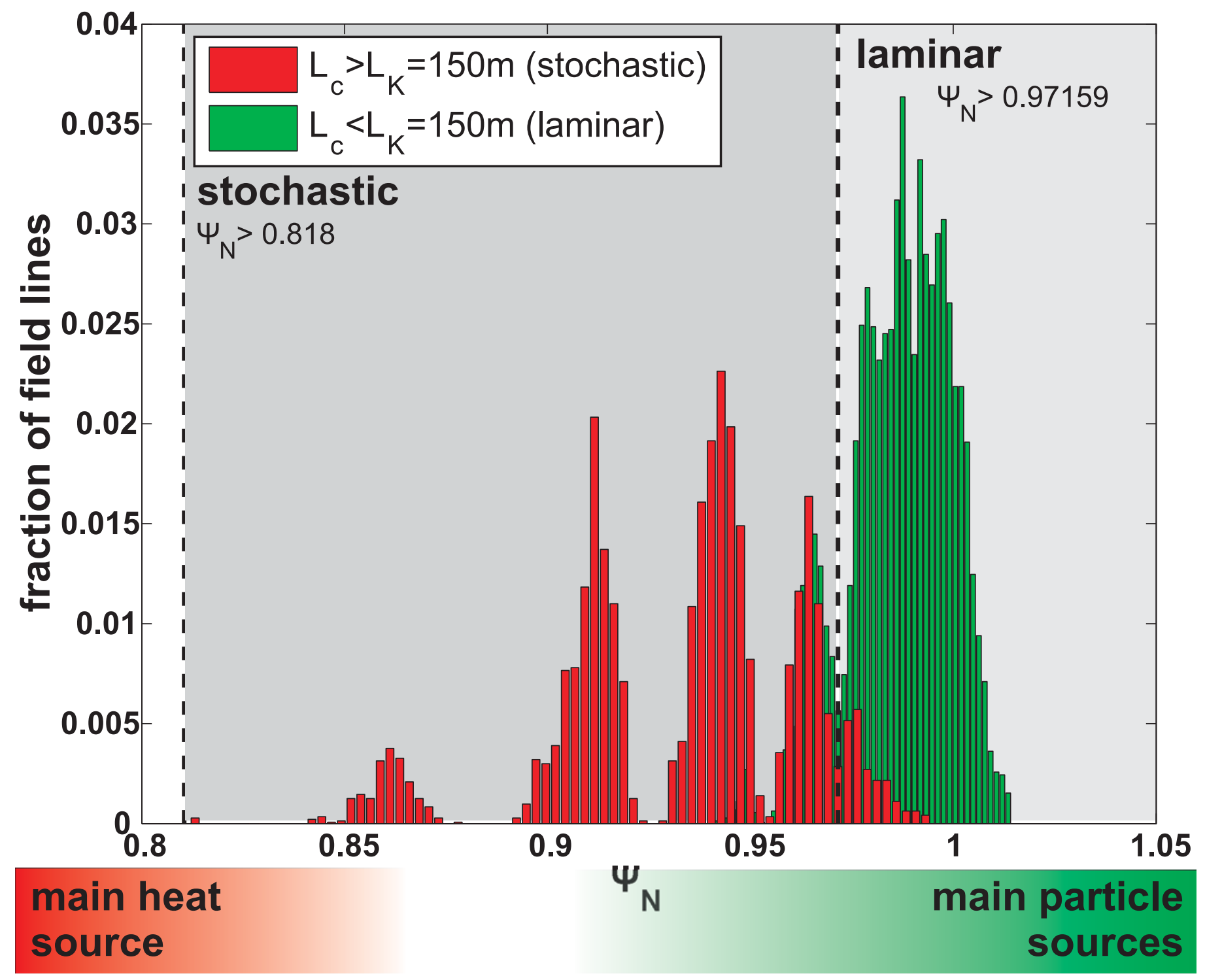



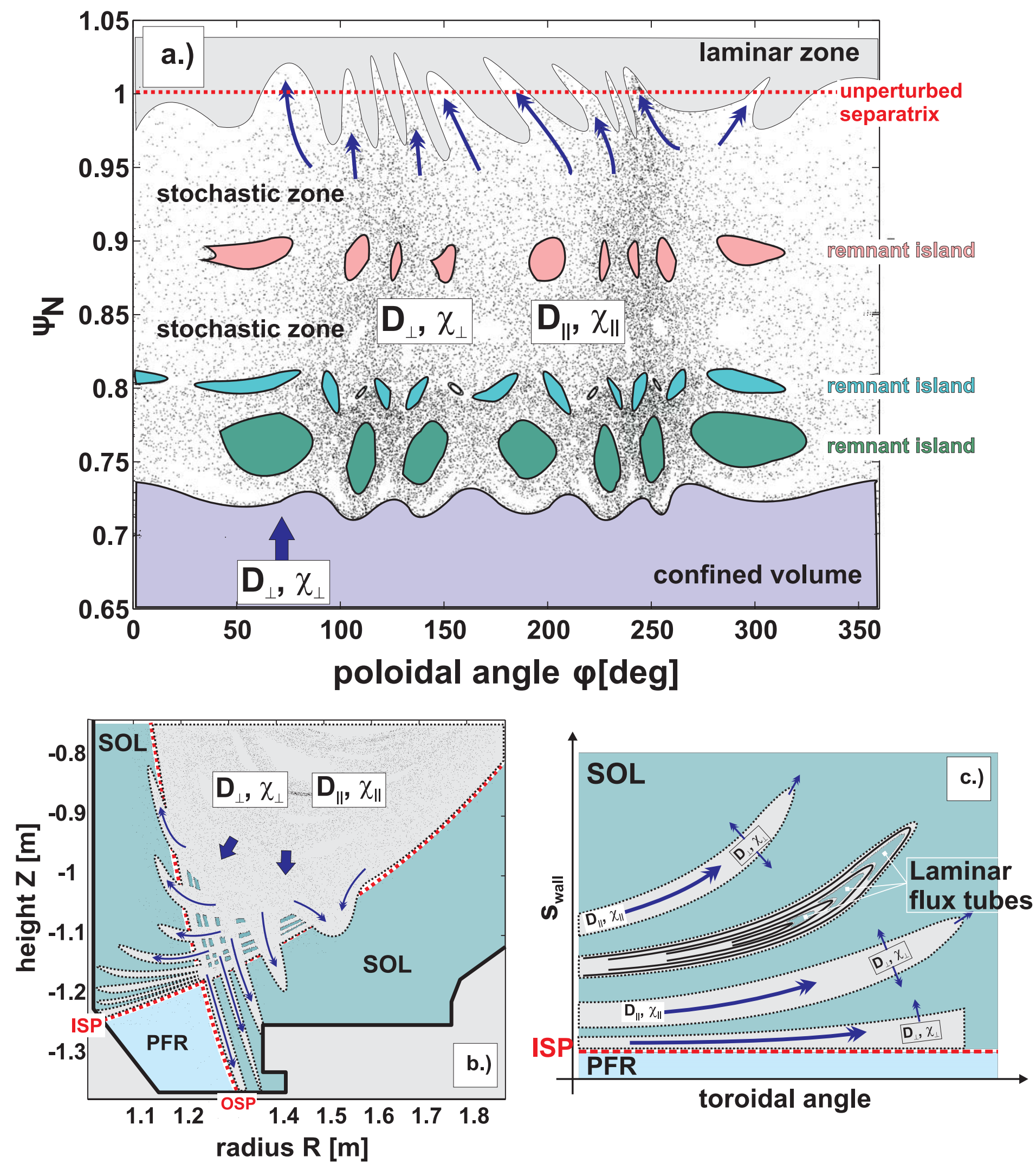


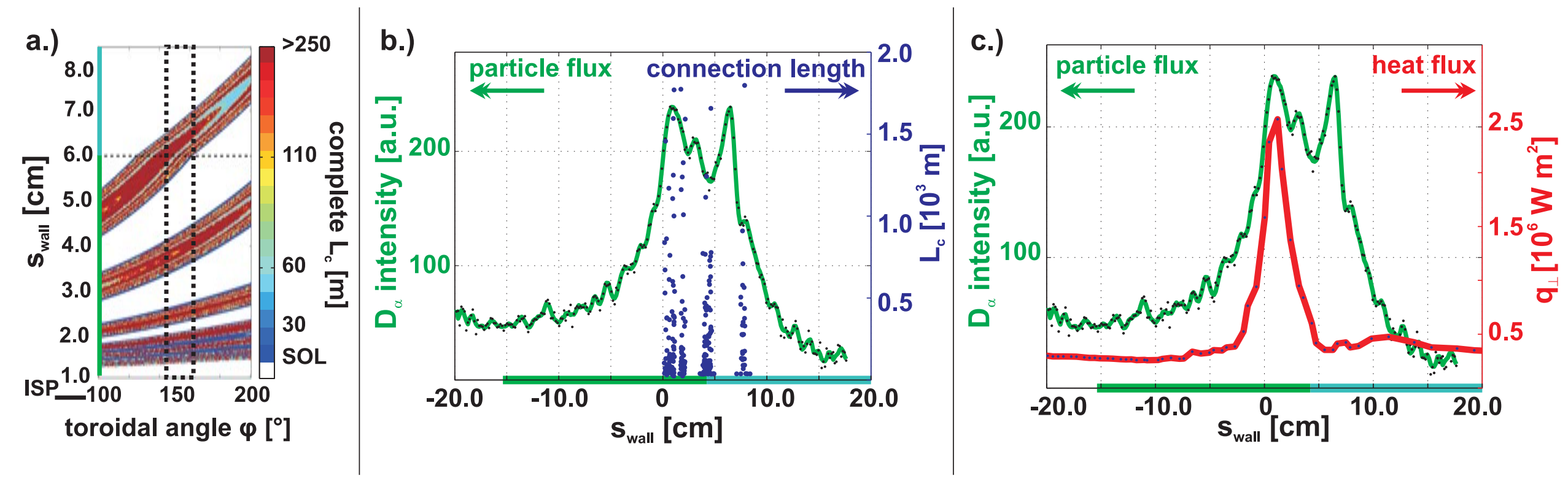



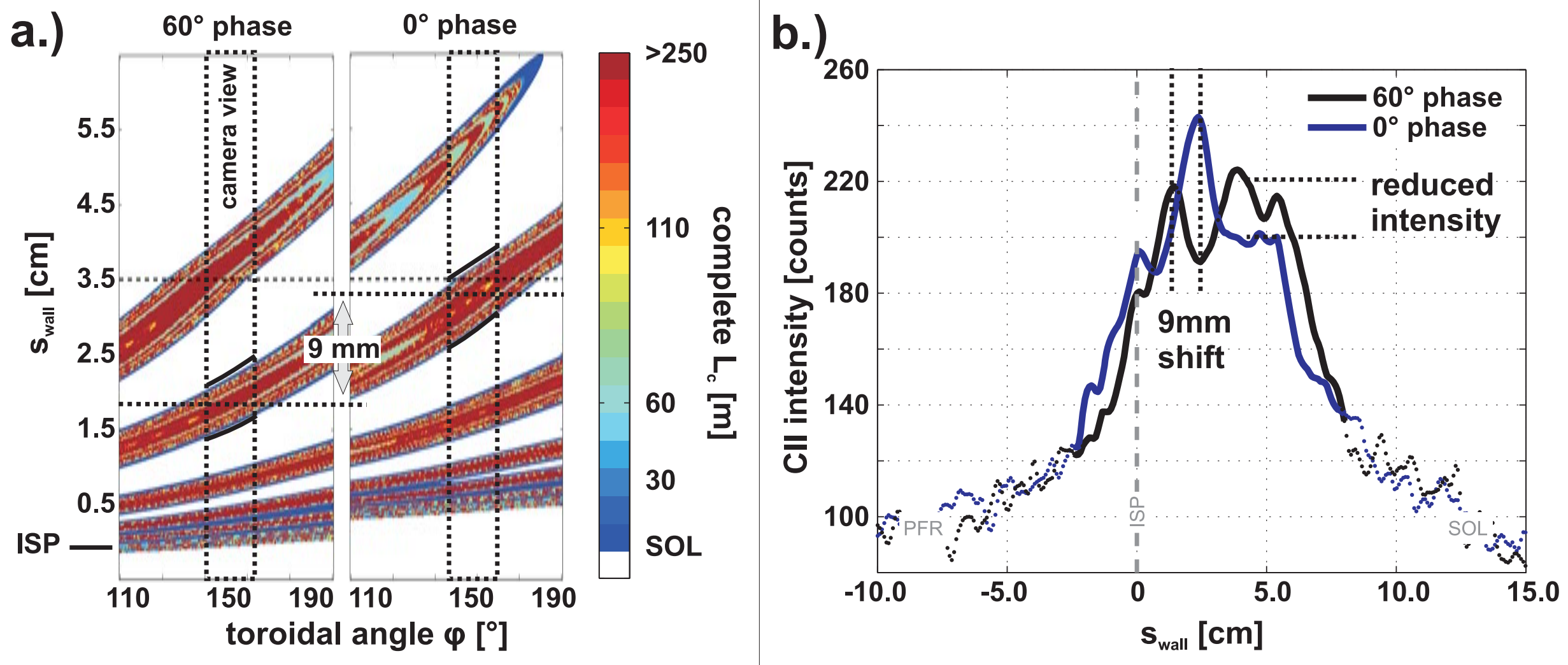

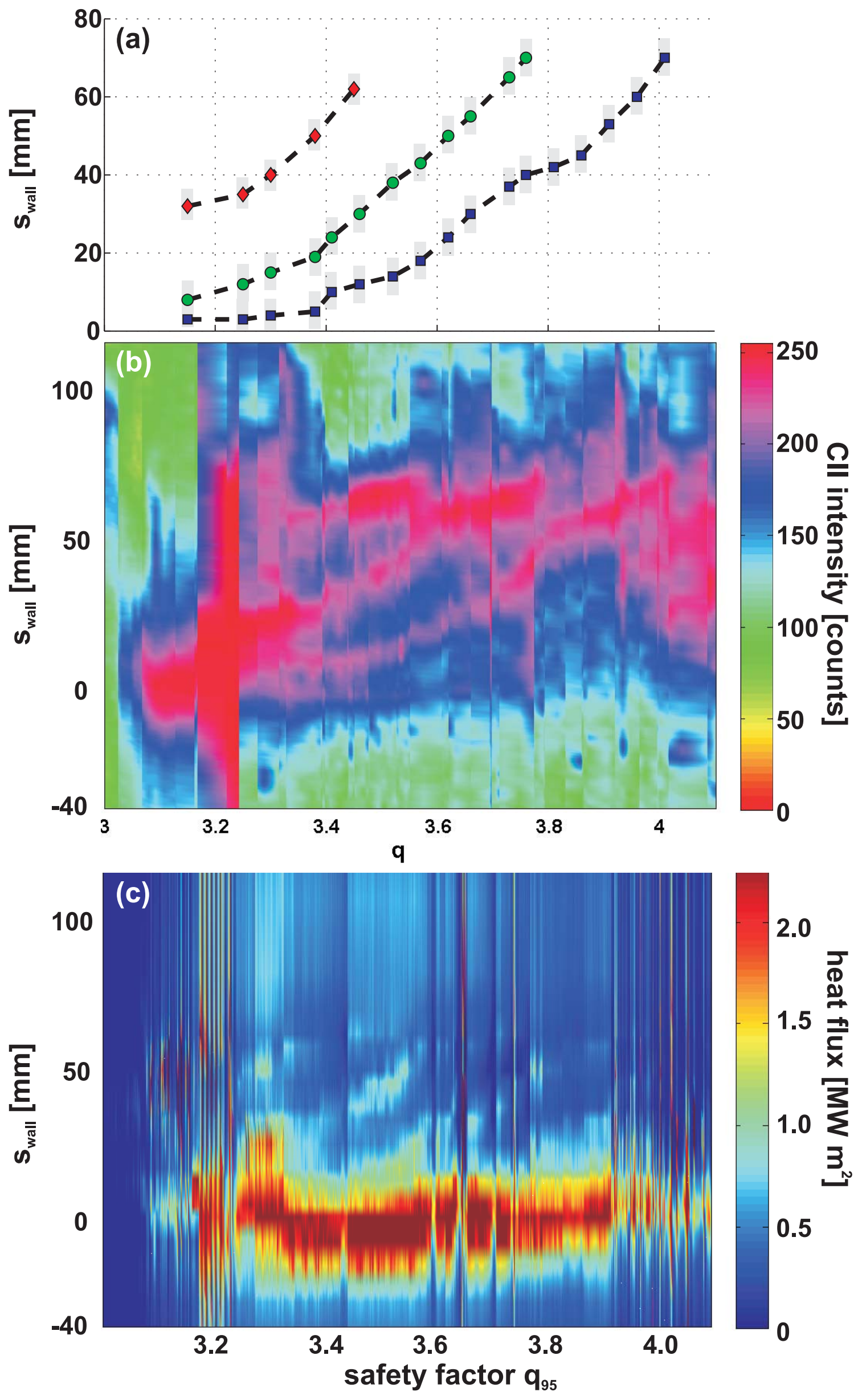

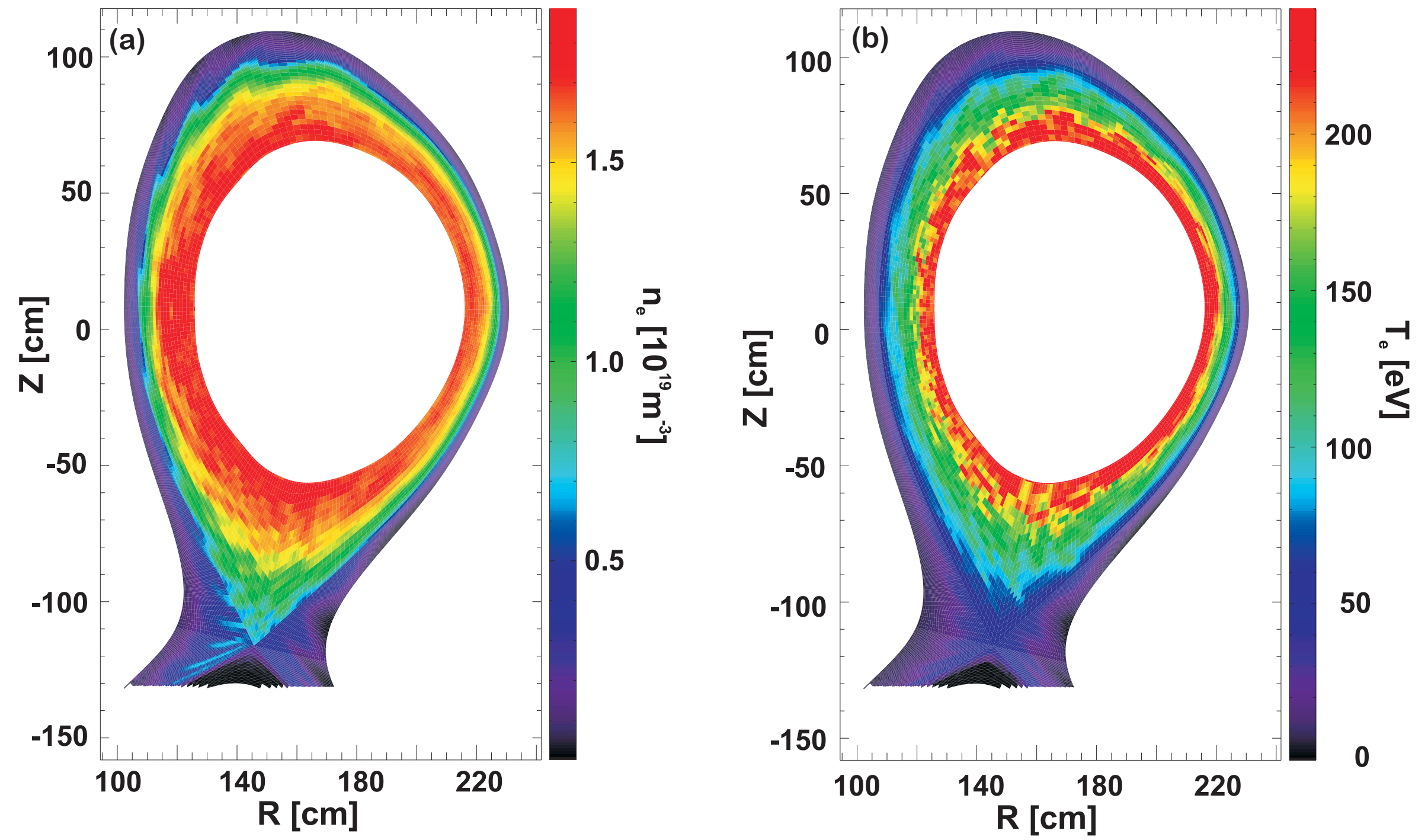


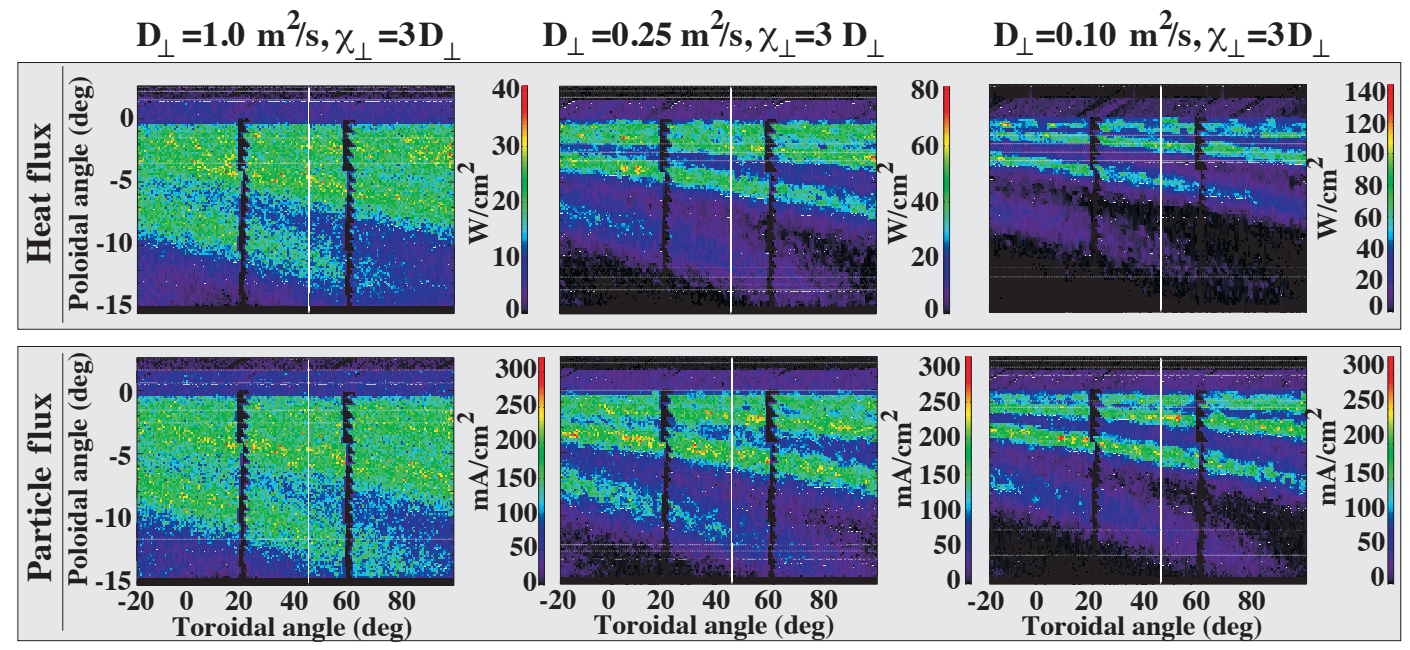

